Study of coincidences between resonant gravitational wave detectors

This article has been downloaded from IOPscience. Please scroll down to see the full text article.

2001 Class. Quantum Grav. 18243

(http://iopscience.iop.org/0264-9381/18/2/304)

View the table of contents for this issue, or go to the journal homepage for more

Download details:

IP Address: 137.138.139.20

The article was downloaded on 05/09/2012 at 10:40

Please note that terms and conditions apply. 


\title{
Study of coincidences between resonant gravitational wave detectors
}

\author{
P Astone ${ }^{1}$, M Bassan ${ }^{2}$, P Bonifazi ${ }^{3}$, P Carelli $^{4}$, E Coccia $^{2}$, C Cosmelli $^{5}$, \\ S D'Antonio ${ }^{5}$, V Fafone ${ }^{6}$, G Federici ${ }^{1}$, A Marini ${ }^{6}$, Y Minenkov ${ }^{2}$, \\ I Modena $^{2}$, G Modestino ${ }^{6}$, A Moleti ${ }^{2}$, G V Pallottino ${ }^{5}$, G Pizzella ${ }^{7}$, \\ L Quintieri ${ }^{6}$, F Ronga $^{6}$, R Terenzi ${ }^{3}, \mathbf{M ~ V i s c o ~}^{3}$ and $L$ Votano $^{6}$
}

${ }^{1}$ Istituto Nazionale di Fisica Nucleare INFN, Rome, Italy

${ }^{2}$ University of Rome 'Tor Vergata' and INFN, Rome, Italy

${ }^{3}$ IFSI-CNR and INFN, Frascati, Italy

${ }^{4}$ University of L'Aquila and INFN, Rome, Italy

${ }^{5}$ University of Rome 'La Sapienza' and INFN, Rome, Italy

${ }^{6}$ Istituto Nazionale di Fisica Nucleare INFN, Frascati, Italy

${ }^{7}$ University of Rome 'Tor Vergata' and INFN, Frascati, Italy

Received 26 September 2000, in final form 2 November 2000

\begin{abstract}
Coincidences are searched for with the cryogenic resonant gravitational wave detectors EXPLORER and NAUTILUS, during a period of about six months (2 June-14 December 1998) for a total measuring time of $94.5 \mathrm{~d}$, with the purpose of studying new analysis algorithms, based on the physical characteristics of the detectors.
\end{abstract}

PACS numbers: 0480,0430

\section{Introduction}

After the initial experiments with room-temperature resonant detectors, the new generation of cryogenic gravitational wave (GW) antennas entered long-term data taking operation in 1990 (EXPLORER [1]), in 1991 (ALLEGRO [2]), in 1993 (NIOBE [3]), in 1994 (NAUTILUS [4]) and in 1997 (AURIGA [5]).

Recently, an analysis of the data taken in coincidence among all cryogenic resonant detectors in operation during the years 1997 and 1998 has been performed [6]. No coincidence excess was found above background using the event lists produced under the protocol of the International Gravitational Event Collaboration (IGEC), among the groups of ALLEGRO, AURIGA, EXPLORER/NAUTILUS and NIOBE. The coincidence search was done without any particular data selection. However, one can consider the possibility to search for coincidences with events selected according to various possible criteria using all available information (we mention criteria based on the event energy, the event duration, the applied threshold, the shape of the events, the coincidence window, the direction of possible GW and the noise). 
Here we have used algorithms based on physical characteristics of the detectors, such as the event energy (with a new algorithm) and the directionality. In this paper we explore their effect on the coincidence search.

For this purpose we shall use IGEC data obtained from 2 June 1998 when NAUTILUS, after a stop for instrumental improvements, resumed operation. We search for coincidences between NAUTILUS and EXPLORER, whose apparatuses differ only in the operating temperatures ( 0.15 and $2.6 \mathrm{~K}$, respectively) and, in particular, have identical readout systems. Extension of the methods we develop here to other detectors in operation during the same period of time is envisaged.

We are well aware that any data selection jeopardizes the possibility to express the results by means of a probability that a coincidence excess, if any, had been accidental. With this proviso we shall still use parameters obtained from probability estimations for comparing different situations.

\section{Events and signals}

We now briefly describe how we obtain events from the measurements. For EXPLORER and NAUTILUS, whose main characteristics are given in table 1, the data are sampled at intervals of $4.54 \mathrm{~ms}$ and are filtered with a filter matched to short bursts [7] for the detection of delta-like signals. The filter makes use of power spectra obtained with off-line analysis. After the filtering of the raw data, events are extracted as follows. $x(t)$ is the filtered output of the detector. This quantity is normalized, using the detector calibration, such that its square gives the energy innovation $E$ of the oscillation for each sample, expressed in kelvin. For well behaved noise due only to the thermal motion of the bar and to the electronic noise of the amplifier, the distribution of $x(t)$ is normal with zero mean. The variance (average value of the square of $x(t))$ is called the effective temperature and denoted by $T_{\text {eff }}$. The distribution of $x(t)$ is

$$
f(x)=\frac{1}{\sqrt{2 \pi T_{e f f}}} \mathrm{e}^{-x^{2} / 2 T_{e f f}} .
$$

For extracting events we set a threshold in terms of a critical ratio defined by

$$
C R=\frac{|x|-|\bar{x}|}{\sigma(|x|)}=\frac{\sqrt{S N R}-\sqrt{2 / \pi}}{\sqrt{1-2 / \pi}}
$$

where $\sigma(|x|)$ is the standard deviation of $|x|$ (the moving averages $|\bar{x}|$ are taken over the preceding $10 \mathrm{~min}$ ) and

$$
S N R=\frac{E}{T_{e f f}} .
$$

\begin{tabular}{|c|c|c|c|c|c|c|}
\hline Detector & Latitude & Longitude & Orientation & $\begin{array}{l}\text { Mass } \\
(\mathrm{kg})\end{array}$ & $\begin{array}{l}\text { Frequencies } \\
(\mathrm{Hz})\end{array}$ & $\begin{array}{l}\text { Temperature } \\
(\mathrm{K})\end{array}$ \\
\hline EXPLORER & $46.45 \mathrm{~N}$ & $6.20 \mathrm{E}$ & $39^{\circ} \mathrm{E}$ & 2270 & $\begin{array}{l}904.7 \\
921.3\end{array}$ & 2.6 \\
\hline NAUTILUS & $41.82 \mathrm{~N}$ & $12.67 \mathrm{E}$ & $44^{\circ} \mathrm{E}$ & 2270 & $\begin{array}{l}906.97 \\
922.46\end{array}$ & 0.15 \\
\hline
\end{tabular}

Table 1. Main characteristics of the two detectors 


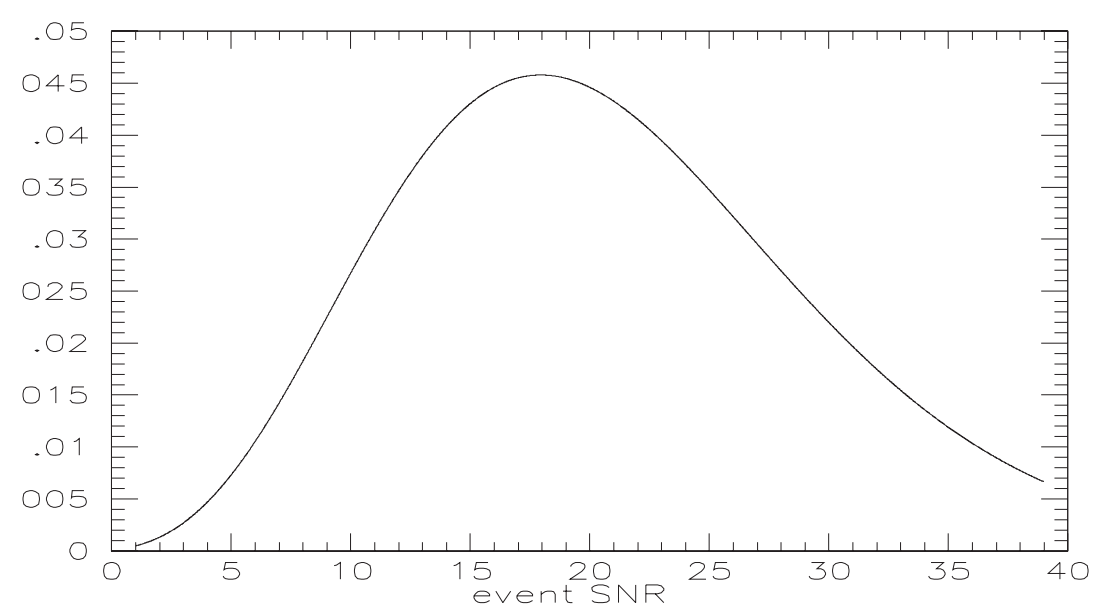

Figure 1. Differential probability that the event has the signal-to-noise ratio shown on the abscissa when the signal has $S N R_{S}=20$.

The threshold is set at $C R=6$ in order to obtain, in the presence of thermal and electronic noise alone, about 100 events per day, as agreed among the partners of the IGEC. This threshold corresponds to an energy $E_{t}=19.5 T_{\text {eff }}$. When $|x|$ goes above the threshold, its time behaviour is considered until it goes below the threshold for more than $10 \mathrm{~s}$. The maximum amplitude and its occurrence time define the event.

In general, the event is due to a combination of a signal which, in the absence of noise, has energy $E_{s}$ (due to GW or other forces) and noise. The theoretical probability to detect a signal with a given $S N R_{S}=E_{s} / T_{\text {eff }}$, in the presence of a well behaved Gaussian noise, is calculated as follows. We put $y=(s+x)^{2}$ where $s \equiv \sqrt{S N R_{s}}$ is the signal we are looking for and $x$ is the Gaussian noise. We obtain easily [8]

$$
\operatorname{probability}\left(S N R_{S}\right)=\int_{S N R_{t}}^{\infty} \frac{1}{\sqrt{2 \pi y}} \mathrm{e}^{-\left(S N R_{s}+y\right) / 2} \cosh \left(\sqrt{y S N R_{S}}\right) \mathrm{d} y
$$

where we put $S N R_{t}=E_{t} / T_{\text {eff }}=19.5$ for the present EXPLORER and NAUTILUS detectors.

The behaviour of the integrand is shown in figure 1. This figure shows the spread of the event energy due to noise for a given $S N R_{s}$ of the applied signal. The distinction between the two concepts, signal and event, is essential for the analysis we propose in this paper.

\section{Data selection}

All the events which are in coincidence within a time window of $\pm 5 \mathrm{~s}$ with events produced by a seismometer are eliminated (about $8 \%$ of the events).

It has been noticed that the experimental data are affected by noise which, in some cases, cannot be observed with any other auxiliary detector. Thus a strategy is needed for deciding when the measurements are considered to be good for the search for coincidences.

We are well aware that the selection of the experimental data must be done with great care and the safest strategy is to establish rules before even looking at the data. We have decided to take into consideration all the data recorded by the detectors (except those vetoed by the seismometer) and accept only the events for which the corresponding $T_{\text {eff }}$ is below a certain threshold. This threshold must be such that we are confident that no signal is being thrown away. All and only the events which have $T_{\text {eff }} \leqslant 100 \mathrm{mK}$ (over the preceding $10 \mathrm{~min}$ ) are 

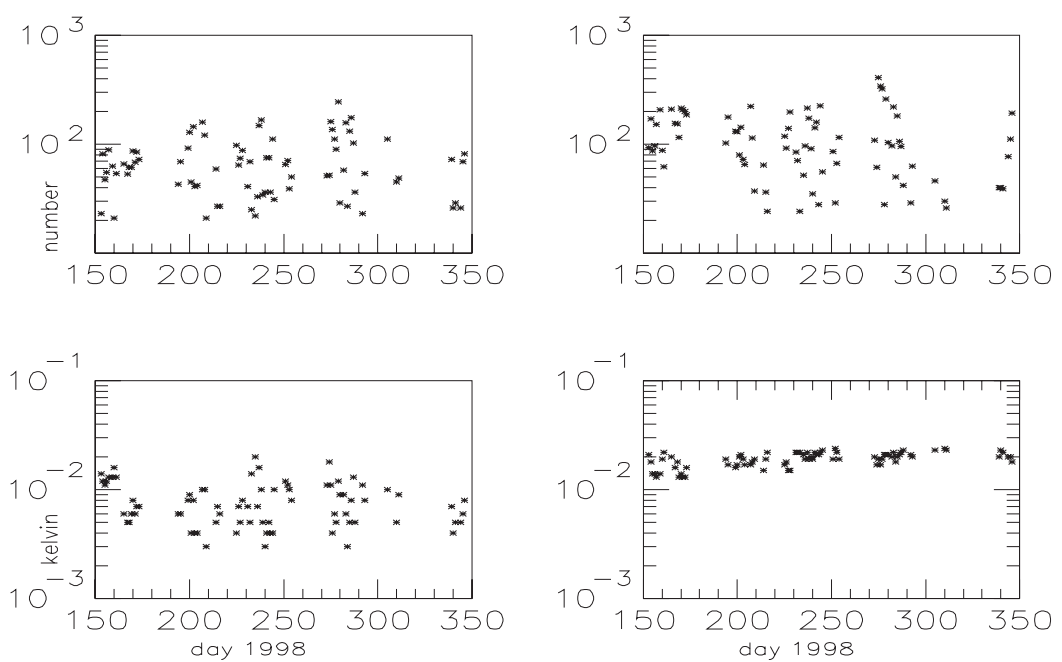

Figure 2. $T_{\text {eff }} \leqslant 25 \mathrm{mK}$. The upper two figures show the number of events/day for NAUTILUS (left) and EXPLORER (right). The lower two figures show the noise temperature $T_{\text {eff }}$ (kelvin), respectively, for NAUTILUS and EXPLORER, daily averaged over the events.

taken into consideration. The events for which the corresponding $T_{\text {eff }}$ is greater than $100 \mathrm{mK}$ are certainly generated at times when the detector is not operating properly.

The following information is available on the IGEC web page for each event:

- time (UT) of the maximum of the event: year, month, day, minute, second;

- $H_{0}$ : bilateral Fourier amplitude at resonance of the maximum;

- SNR: signal-to-noise ratio of the amplitude;

- $T_{\text {eff }}$ : effective temperature $(\mathrm{K})$ of the previous $10 \mathrm{~min}$;

- duration $L$ of the event, in number of samples (4.54 ms);

- time in seconds between the beginning and the maximum of the event.

The relationship between the Fourier transform $H_{0}$ of the event amplitude and the energy $E$ of the event is given by [9]

$$
H_{0}=7.97 \times 10^{-21} \sqrt{E}
$$

with $H_{0}$ in units of $\mathrm{Hz}^{-1}$ and $E$ in kelvin.

Looking at these events we have noticed that some events occur during periods of high disturbance. Since we are elaborating here strategies for data analysis, we thought it convenient to select the data to be used in our analysis in various ways according to the noise. Thus another way of choosing the data to be analysed is to select periods with smaller noise. We apply two more data selections, only events with $T_{\text {eff }} \leqslant 50 \mathrm{mK}$ for both EXPLORER and NAUTILUS and only events with $T_{\text {eff }} \leqslant 25 \mathrm{mK}$. These data selection has been applied by us in a previously published paper [10]. In figure 2 we show the number of events per day and the hourly averages of $T_{\text {eff }}$ for EXPLORER and NAUTILUS for the case of $T_{e f f} \leqslant 25 \mathrm{mK}$.

We note large fluctuations, in spite of the stringent criteria for the data selection. Information on the various data selections are given in table 2 . We note that the number of available hours of measurement becomes rather small when lowering the threshold for $T_{e f f}$, so that any possible result becomes statistically weaker at low $T_{\text {eff }}$. 
Table 2. Total number of events, $N$, number of hours of data taking, average noise temperature $\left\langle T_{e f f}\right\rangle$ and hours in common, when both detectors were operating simultaneously.

\begin{tabular}{lllllll}
\hline & $\begin{array}{l}T_{\text {eff }} \\
(\mathrm{mK})\end{array}$ & $N$ & $\mathrm{~h}$ & $\begin{array}{l}\left\langle T_{\text {eff }}\right\rangle \\
(\mathrm{mK})\end{array}$ & $\begin{array}{l}\text { Hours } \\
\text { in common }\end{array}$ & $N$ \\
\hline EXPLORER & $\leqslant 100$ & 55070 & 3415 & 40.6 & 2271 & 37944 \\
NAUTILUS & & 37734 & 3450 & 19.1 & & 24118 \\
EXPLORER & $\leqslant 50$ & 39211 & 2759 & 28.9 & 1816 & 26481 \\
NAUTILUS & & 34148 & 3371 & 14.0 & & 16677 \\
EXPLORER & $\leqslant 25$ & 16172 & 1498 & 18.7 & 931 & 9765 \\
NAUTILUS & & 27823 & 3168 & 9.3 & & 5999 \\
\hline
\end{tabular}

\section{Searching for coincidences}

For the search for coincidences it is important to establish the time window. We have decided to adopt the same window used in past analyses, in particular that described in [6,11], $w= \pm 1 \mathrm{~s}$. This is a reasonable choice considering the bandwidth of present detectors (of the order of $1 \mathrm{~Hz}$ ) and some time inaccuracy.

As is well known, the analysis in a coincidence search consists essentially in comparing the detected coincidences at zero time delay with the background, that is with coincidences occurring by chance. In order to measure the background due to the accidental coincidences, using a procedure adopted since the beginning of the gravitational wave experiments [12], we have shifted the time of occurrence of the events of one of the two detectors 1000 times in steps of $2 \mathrm{~s}$, from $-1000 \mathrm{~s}$ to $+1000 \mathrm{~s}$. For each time shift we get a number of coincidences. If the time shift is zero we get the number $n_{c}$ of real coincidences. The background is calculated from the average number of the $n_{\text {shift }}$ accidental coincidences obtained from the 1000 time shifts

$$
\bar{n}=\frac{\sum_{1}^{1000} n_{\text {shift }}}{1000} .
$$

With this experimental procedure for the evaluation of the background we circumvent the problems arising from a not very stationary distribution of events, provided we test the distribution of the shifted coincidences properly (see figure 4 and [13]).

The result of our search for coincidences is given in table 3. There is no coincidence excess between EXPLORER and NAUTILUS, even for selected periods with smaller noise.

Table 3. The number of coincidences, $n_{c}$, and the average number of accidentals, $\bar{n}$. The total period of time in common when $T_{\text {eff }} \leqslant 100 \mathrm{mK}$ is $94.5 \mathrm{~d}$.

\begin{tabular}{lrrr}
\hline$T_{\text {eff }}$ & $n_{c}$ & \multicolumn{1}{c}{$\bar{n}$} & Hours \\
\hline$\leqslant 100 \mathrm{mK}$ & 223 & 231.7 & 2271 \\
$\leqslant 50 \mathrm{mK}$ & 137 & 139.8 & 1816 \\
$\leqslant 25 \mathrm{mK}$ & 32 & 36.2 & 931 \\
\hline
\end{tabular}

\section{Data selection using the event energy}

We now want to apply data selection algorithms based on the event energy. The most obvious one is to search for pairs of events which have (approximately) the same energy. In the past 
this energy criterion has been applied, requiring that the responses of the EXPLORER detector at the two resonance modes were within a factor of two from each other [1]. Later we realized that the effect of the noise on signals near threshold is such that the event energies are only lightly correlated to the signal energies [14], and this reduces the efficiency of algorithms based on the event energy.

Recently, an important result was found [15]. It has been seen that the distribution of the energy ratios of the event energies of two detectors, in the case of non-Gaussian noise, is different for real coincidences and accidental coincidences. This has pushed us to reconsider the importance of applying selection algorithms based on the event energies.

To make use of the event energy, in particular with detectors with different sensitivities, we must consider the result shown in figure 1 which indicates the chance of having a certain event energy for a given signal energy. In principle, all event energies are possible, from zero to infinity. Our procedure here is to consider only event energies within \pm one sigma from the signal energy (that is, we consider events included in $68 \%$ of the area under the curve in figure 1).

We do not know the signal energy. The new algorithm we propose is the following. We consider signals over a wide range, say $E_{s}$ from $20 \mathrm{mK}$ to $2 \mathrm{~K}$ in steps of $20 \mathrm{mK}$. We find the coincident events, at zero delay (the real coincidences) and at shifted times (for the estimation of the accidentals). For each assumed signal with energy $E_{S}$ we calculate the $S N R_{S}$ different for each event, since the noise $T_{\text {eff }}$ depends on the detected event and it is also different for the two detectors. We then verify whether the $S N R_{\text {event }}$ falls into $S N R_{S} \pm 1 \sigma$, having calculated for each $S N R_{s}$ the probability curve like that shown in figure 1 for $S N R_{S}=20$. If the two event energies are compatible with the event energy expected for any of the assumed signals then we accept the coincidence (real or shifted).

The result of this analysis is given in table 4 . We notice that the use of the energy selection algorithm has reduced the number of accidental coincidences by a factor of three.

Table 4. Energy algorithm. Number $n_{c}$ of coincidences, average number $\bar{n}$ of accidentals and the covered time period for the three data selection.

\begin{tabular}{lllr}
\hline$T_{\text {eff }}$ & $n_{c}$ & $\bar{n}$ & Hours \\
\hline$\leqslant 100 \mathrm{mK}$ & 61 & 50.5 & 2271 \\
$\leqslant 50 \mathrm{mK}$ & 45 & 37.7 & 1816 \\
$\leqslant 25 \mathrm{mK}$ & 11 & 10.3 & 931 \\
\hline
\end{tabular}

\section{Event selection according to the detector orientation with respect to the galactic centre}

No extragalactic GW signals should be detected with the present detectors. Therefore, we shall focus our attention on possible sources located in the Galaxy. If any of these sources exist we should expect a more favourable condition of detection when the detectors are oriented with their axes perpendicular to the direction toward the galactic centre (GC), since, the bar cross section is proportional to $\sin ^{4}(\theta)$, where $\theta$ is the angle between the detector axis and the direction to the GC.

After having applied the above energy algorithm, we search for coincidences considering only events obtained when the detectors were oriented with $\theta$ greater than various given values and, according to the previous sections, for the various data selection. 

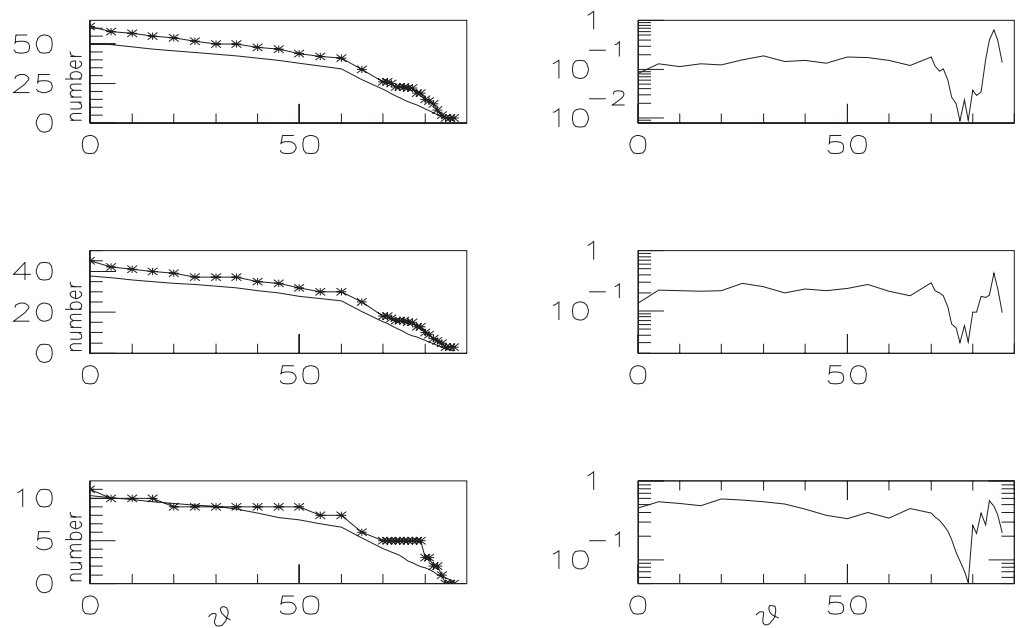

Figure 3. The upper left-hand figure shows (versus $\theta$ and for $T_{\text {eff }} \leqslant 100 \mathrm{mK}$ ) the integral number of coincidences $n_{c}$ (indicated with asterisks) and the average number of accidentals $\bar{n}$ (calculated from the number of coincidences at zero delay and the average background $\bar{n}$ measured with 1000 delays). The right-hand figure shows the Poisson probability that the observed number of coincidences $n_{c}$ was due to a background fluctuation. Similarly, the second line of figures refers to the data selection $T_{\text {eff }} \leqslant 50 \mathrm{mK}$ and the third line to the data selection $T_{\text {eff }} \leqslant 25 \mathrm{mK}$.

The result is given in figure 3 and shows a larger coincidence excess when the detector axes tend to be perpendicular to the direction towards the GC. Above $\theta \sim 79^{\circ}$ the number $n_{c}$ of coincidences drops quickly. If not instrumental, the quick drop could be taken being as due to the width of the source. The time spent by the detectors when $\theta \geqslant 79^{\circ}$ is $20 \%$ of the total time of $94.5 \mathrm{~d}$.

We want now to verify that the evaluation of the background is done properly. We do this with the condition of greatest coincidence excess, that is for $\theta \geqslant 79^{\circ}$. We must consider that by selecting only times when the detectors had certain orientations we have several empty time regions. This makes it possible that in doing the shifting operation to evaluate the background one uses time periods of different duration. We have determined these time periods and found that they vary by a few per cent, with a maximum of $-10 \%$ for a time shift of $+1000 \mathrm{~s}$. In figure 4 we show, for the case $\theta \geqslant 79^{\circ}$ and $T_{\text {eff }} \leqslant 100 \mathrm{mK}$, the delay histogram with no correction and the delay histogram corrected for the different periods of time for each shift. In this particular case the correction applies for a very small amount, only for delays greater than about $700 \mathrm{~s}$.

\section{Conclusions}

In order to make a first step towards a complete analysis, we have selected the IGEC events of EXPLORER and NAUTILUS using algorithms based on known physical characteristics of the detectors. In particular, a new algorithm which makes use of the event energy has been devised.

With event selection based on this algorithm we find an excess of coincidences at zero time delay in the direction of the galactic centre. As is well known in the scientific community, no GW signals are expected to be observed with the present detector sensitivity. Since this result would open new possibilities, a careful Bayesian approach suggests that, given the Poisson 

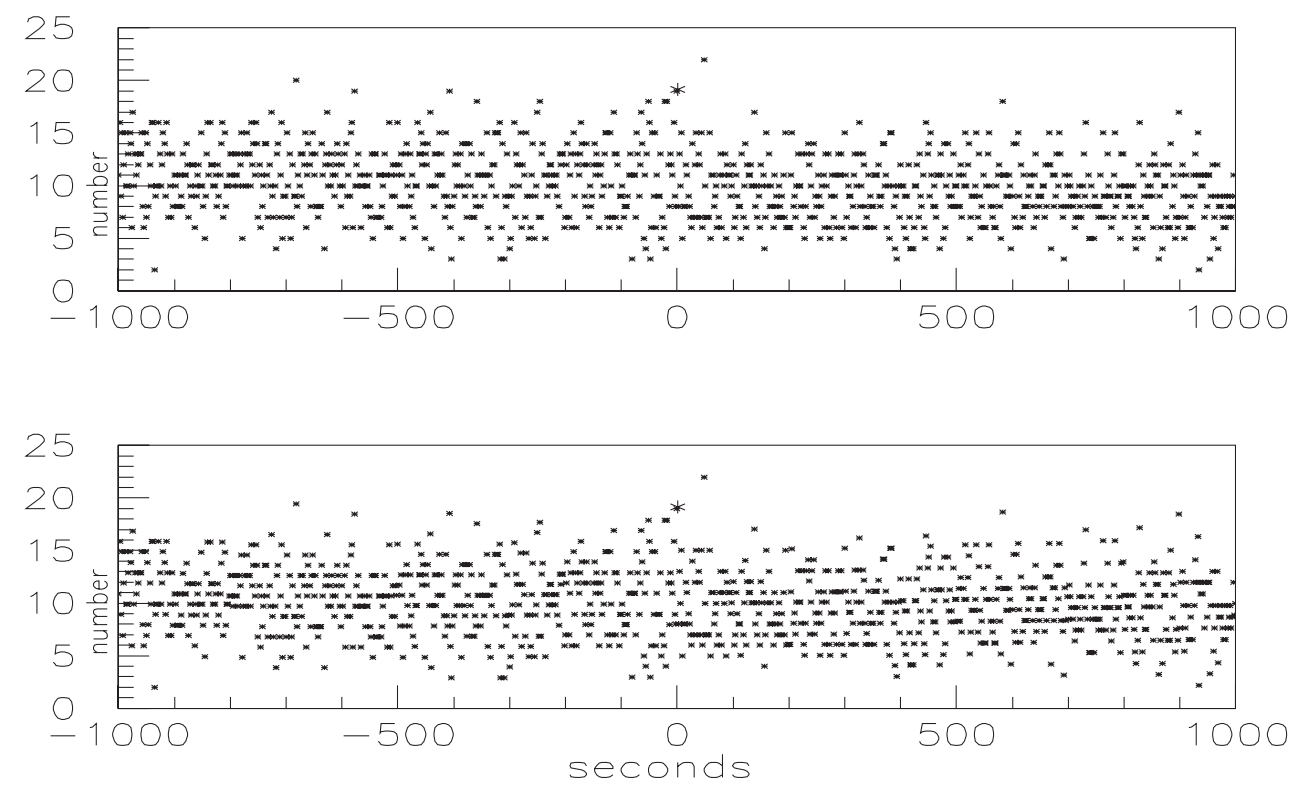

Figure 4. Data selection with $T_{\text {eff }} \leqslant 100 \mathrm{mK}$. In the upper figure we show, for $\theta \geqslant 79^{\circ}$, the delay histogram. In the lower figure we show the same data normalized for the duration of the time period used for each time shift. In this particular case the normalization turns out to be very small, almost barely visible for delays above $700 \mathrm{~s}$, but it is worth noting that a possible effect due to different time coverages at various delays has been taken into account. The large asterisks indicate the 19 coincidences at zero time delay.

probabilities of a few per cent, new data with other detectors are required before we can ensure that GW from the GC have been indeed observed. Thus, at present, we feel that the coincidence excess is not large enough to establish a claim for detection of true signals, but it is important information to make available to the scientific community. We believe that the procedures adopted here might be useful for detecting gravitational waves with more or better data.

\section{Acknowledgments}

We thank W O Hamilton and W W Johnson for discussions and suggestions. We thank the European Center for Nuclear Physics (CERN) for hospitality and for the supply of cryogenic liquids. We thank F Campolungo, R Lenci, G Martinelli, E Serrani, R Simonetti and F Tabacchioni for precious technical assistance.

\section{References}

[1] Astone P et al 1993 Phys. Rev. D 47362

[2] Mauceli E et al 1996 Phys. Rev. D 541264

[3] Blair D G et al 1995 Phys. Rev. Lett. 741908

[4] Astone P et al 1997 Astroparticle Phys. 7231

[5] Cerdonio M et al 1994 1st Edoardo Amaldi Conf. on Gravitational Wave Experiments (Frascati, 14-7 June)

[6] Initial operation of the IGEC Collaboration Prodi G A et al 4th Gravitational Wave Data Analysis Workshop (GWDAW 99) (Rome, 2-4 December 1999)

Prodi G A et al 2000 Int. J. Mod. Phys. D 9237

(Prodi G A et al 2000 Preprint astro-ph/0003106) 
[7] Astone P, Buttiglione C, Frasca S, Pallottino G V and Pizzella G 1997 Nuovo Cimento 209

[8] Papoulis A 1965 Probability, Random Variables and Stochastic Processes (New York: McGraw-Hill) p 126

[9] Astone P et al 1990 Gravitational Astronomy ed D E McClelland and H A Bachor (Singapore: World Scientific)

[10] Astone P et al 1999 Astroparticle Phys. 1083

[11] Astone P et al 1999 Phys. Rev. D 59122001

[12] Weber J 1969 Phys. Rev. Lett. 221320

[13] Astone P, Frasca S and Pizzella G 1999 Background estimation in a gravitational wave experiment 4th Gravitational Wave Data Analysis Workshop (GWDAW 99) (Rome, 2-4 December 1999)

Astone P, Frasca S and Pizzella G 2000 Int. J. Mod. Phys. D 9341

(Astone P, Frasca S and Pizzella G 2000 Preprint gr-qc/0002004)

[14] Astone P, Pallottino G V and Pizzella G 1998 Gen. Rel. Grav. 30105

[15] Blair D et al 2000 Gen. Rel. Grav. 321281 